\title{
Clip Reconstruction of a Previously Leaked Large Complex Posterior Communicating Segment Aneurysm with Extradural Anterior Clinoidectomy
}

\author{
Duygu Baykal ${ }^{1}$ Burak Ozaydin ${ }^{1} \quad$ Ufuk Erginoglu ${ }^{1}$ Mustafa K. Baskaya ${ }^{2}$
}

${ }^{1}$ Department of Neurological Surgery, University of Wisconsin-Madison

Address for correspondence Mustafa K. Baskaya, MD, Department of School of Medicine and Public Health, Madison, Wisconsin, United States

2 Department of Neurological Surgery, University of WisconsinNeurological Surgery, University of Wisconsin-Madison, 600 Highland Avenue, CSC K8/828, Madison, WI 53792, United States

Madison School of Medicine and Public Health, Madison, Wisconsin, (e-mail: baskaya@neurosurgery.wisc.edu).

United States

J Neurol Surg B Skull Base 2022;83(suppl S3):e606-e607.

\begin{abstract}
Fusiform or near-fusiform aneurysms that involve the long segment of the supraclinoid internal carotid artery (ICA) pose significant challenges to neurovascular surgeons. Involvement of the origin of vital branching arteries in this segment may preclude safe treatment with flow diverting stents. In addition, clip reconstruction may also not be possible in this region due to entire or near-entire involvement of the circumference of the ICA (- Fig. 1). In this video article, we present a case of a complex and previously leaked, (visualized with hemosiderin) aneurysm of the posterior communicating segment of the ICA, in a 60-year-old female. Multiple complexities made this aneurysm challenging to treat. These included (1) a 270-degree encirclement of the ICA with multiple lobulations that left only a small section of nondiseased vessel wall, (2) a relatively short segment of the

\section{Keywords}

- complex aneurysm

- hemosiderin

- anterior clinoidectomy

- fetal posterior communicating artery supraclinoidal ICA that made proximal control challenging thus requiring an extradural anterior clinoidectomy, (3) a fetal posterior communicating artery that originated immediately proximal to the beginning of the aneurysm, and lastly, (4) an anterior choroidal artery that was firmly adherent over the aneurysm dome. In this video, we present the microsurgical steps for dealing with this complex aneurysm, including extradural clinoidectomy and clip reconstruction (-Fig. 2). Postoperatively, the patient woke up without any deficits. Angiography showed complete obliteration of the aneurysm.

The link to the video can be found at: https://youtu.be/3Zz-ecvIDIc.
\end{abstract}

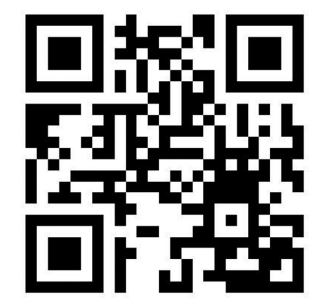

received

April 30, 2020

accepted

October 18, 2020

published online

April 8, 2021

www.thieme.com/skullbasevideos

www.thieme.com/jnlsbvideos

DOI https://doi.org/ 10.1055/s-0041-1726425. ISSN 2193-6331.

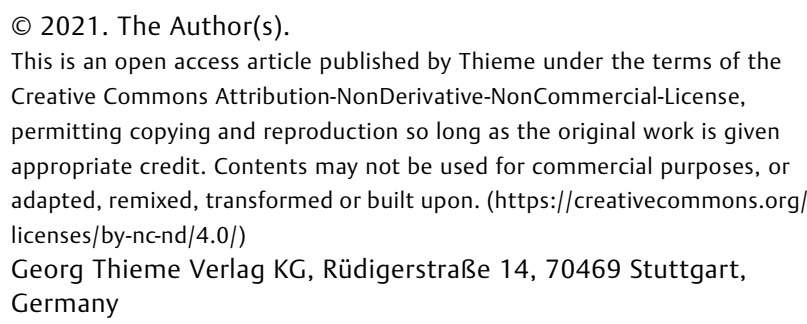




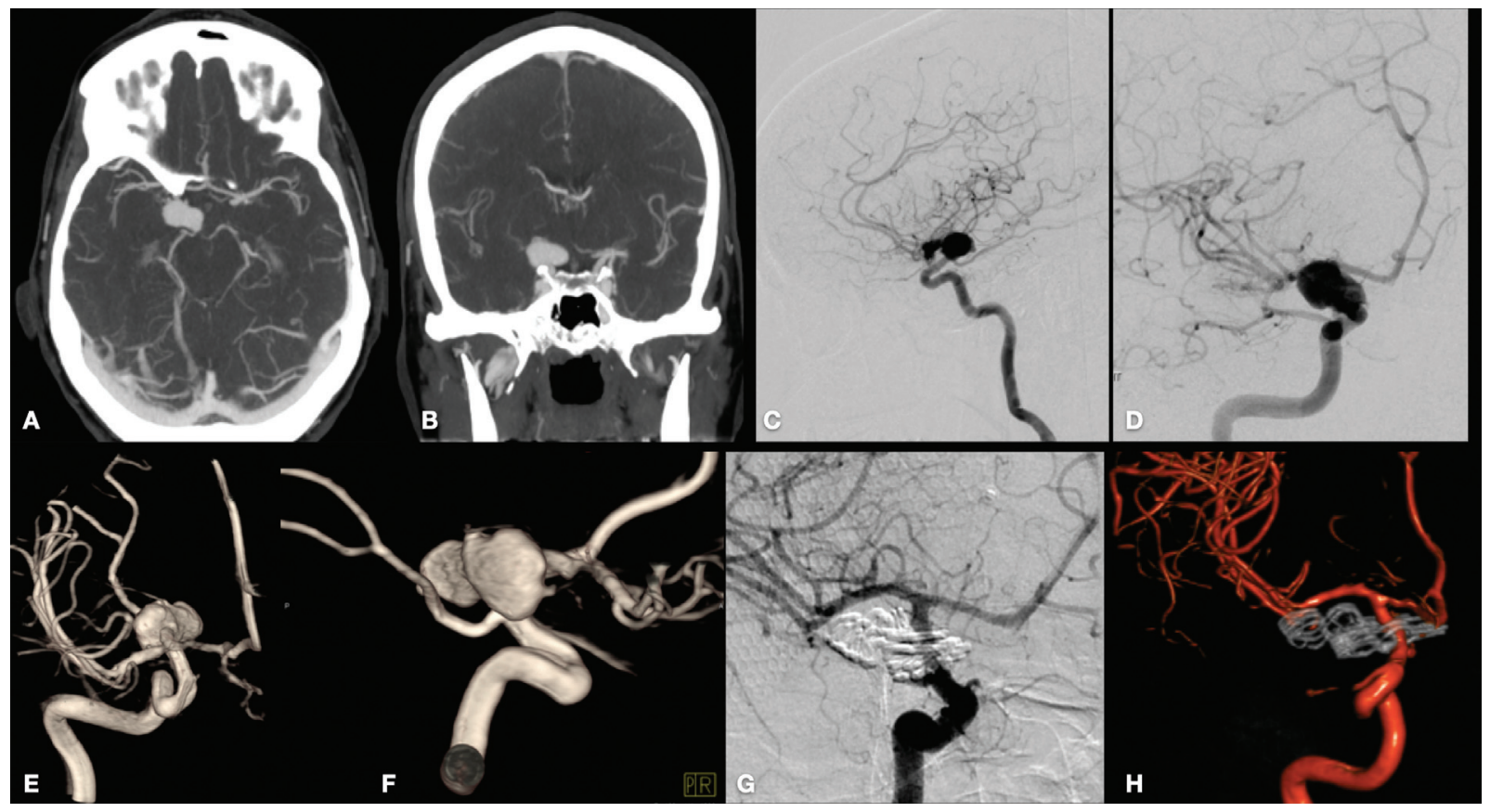

Fig. 1 Preoperative axial (A) and coronal (B) computerized tomography angiography, lateral (C) and right anterior oblique (D) digital subtraction angiography (DSA), and three-dimensional (3D) reconstruction (E, F) show a right-sided, large and complex posterior communicating segment aneurysm of the internal carotid artery. Postoperative right anterior oblique (G), and 3D reconstruction (H) show complete clip obliteration of the aneurysm. 3D, three-dimensional.

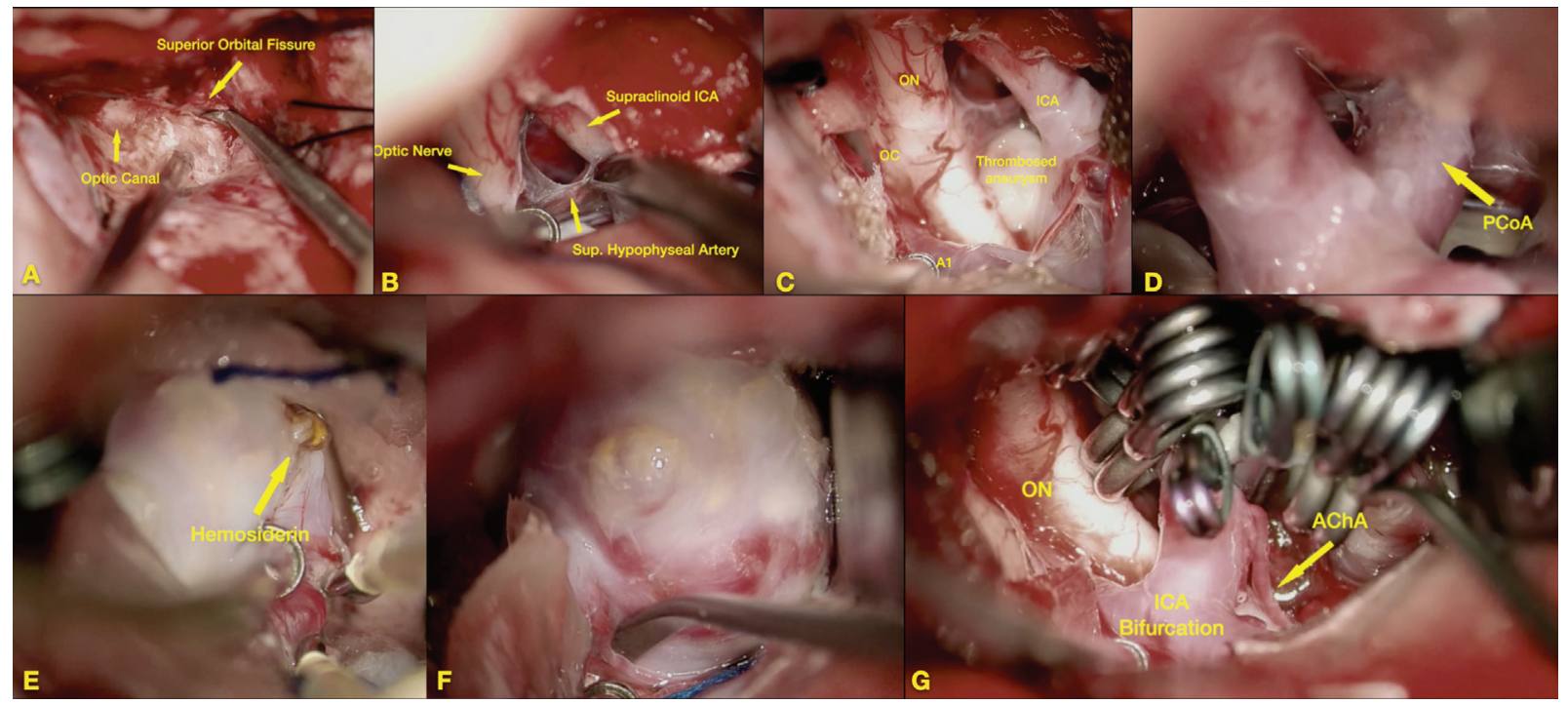

Fig. 2 Intraoperative photomicrographs showing the surgical steps. (A) Extradural anterior clinoidectomy. Note the decompressed optic canal and lateral orbitotomy. (B) Arachnoid dissection of the opticocarotid cistern. Optic nerve, superior hypophyseal artery, and the supraclinoid segment of the internal carotid artery (ICA) is visible. (C) Thrombosed part of the aneurysm is visible after completed arachnoid dissection. Note the optic nerve (ON), optic chiasm (OC), ICA, and A1 segment of the anterior cerebral artery. (D) Fetal posterior communicating artery (PCoA) originating from ICA. (E) Hemosiderin is visible over the aneurysm dome. (F) Adherent anterior choroidal artery (AChA) over the aneurysm dome. Instrument tip is at the AChA. Note the lobular appearance of the aneurysm. (G) View of the completely obliterated aneurysm after multi-clip reconstruction. ON, ICA bifurcation, and untethered AChA is visible.

Conflict of Interest

None declared.

\section{Funding}

The authors have no personal, financial, or institutional interest in any of the drugs, materials, or devices described in this article. 